\title{
NHS information strategy and its impact on child health
}

\author{
Mitch Blair
}

Paediatricians need to know what local and national information strategy is about so that they are included in the debates taking place at both levels and secondly because the possession of relevant and accurate information about the child health services is becoming vital in the fight for a share of limited NHS resources.

The NHS has an Information Management Group as part of the executive which has produced an information management and technology (IM and T) strategy. The basic tenet of the strategy is that patient care is central and the vision is to 'To support better care and communications through appropriate use of IM and T'. The belief is that this should allow better targeting of resources and lead to improvements in efficiency of services. ${ }^{1}$

The thrust of the strategy is to provide person based information systems holding secure and confidential data. Management and administrative data is to be obtained from clinical operational systems (and not the other way round as is often the case at present). The infrastructure would allow information to be shared across the NHS in an integrated way. The vision is eventually to produce an electronic patient record with links between the whole range of hospital and community service providers and internally to support and laboratory services. Included in this strategy is the development of a new NHS number, a central administrative register, a thesaurus of clinical terms, and standards for intercommunication and confidentiality. Some of these developments and their impact on child health services are reviewed below.

\section{A new NHS number}

The NHS number is a unique identifier which is carried by that patient wherever they use the health service whether they live in Truro or Carlisle. At present patients often have a district (Körner) number and one or more hospital numbers in addition to their NHS number. The allocation of a district ID number was one of the recommendations of the Steering Group on Health Services Information chaired by Edith Körner. This committee was set up in 1980, and as part of its remit recommended the use of minimum data sets required for management of the NHS. ${ }^{2}$ The number has no fixed structure and varies across the country. It was designed to track patients within a specific district and not between districts. It is allocated as part of the patient admission data set. In the case of a child this may be at birth or subsequently on admission to the children's ward or when coming to the outpatients department for the first time. The NHS number, however, is allocated whenever a parent registers the birth of an infant with the local registrar of births, deaths, and marriages. Birth registration has been a statutory requirement in England and Wales since 1836 and the onus of registration passed from the registrar to parents in the Births and Deaths Registration Act of $1874 .{ }^{3}$ Registration has to take place within six weeks of the birth and at least $99.5 \%$ of births are registered within this time. Interestingly, first born infants and those born in the north of the country are likely to be registered earlier than those born subsequently or those born in the south (OPCS, NHS Central Register). Once an infant is registered, an NHS number is allocated by the registrar (a computerised process since 1990) and the child can then be formally registered with a local general practitioner. The number is unique to that individual and in fact consists of a combination of letters and numbers mainly for historical reasons - for example, YYA 295 C 106, where YYA refers to the district, 2 to the subdistrict, 95 to year of birth, $C$ to a letter in the registrar's register, and 106 to the number in the register schedule. This is to be replaced in July 1995 by the issue of a new 10 digit NHS number (nine plus a check digit) to all those registered on the central NHS register. There is an estimated $1.5 \%$ duplication rate of NHS numbers, that is more than one number per person. This is more common in immigrants to the UK. There is no duplication of numbers in children and adults born in the UK (OPCS, NHS Central Register).

A unique identifier is an important concept in linking health events to specific individuals and is more reliable than date of birth, name, or address. A local study in Nottingham involving an audit of child health surveillance among 29 general practices has shown a name change rate of $13 \%$ within the first three months and mobility of the family of $10 \%$ making it difficult to track patients for screening tests ( $M$ Blair, unpublished data). A pro-

portion of babies require treatment in hospital 
within the first few days and weeks. With the introduction of person based information systems and the change in the nature of contracting, it will become essential to be able to link the correct treatment programme to the right patient. Several professional groups are lobbying the Department of Health to consider the issuing of the NHS number at birth and not at birth registration.

\section{The NHS administrative register}

This is a basic computerised register shared between all providers, containing name, address, NHS number, and details of the general practitioner. The register will not hold any clinical data. NHS-wide networking of this register will make it easier to track details of patients who have moved around the country or have changed name. This could bring enormous benefits to patients in ensuring that records follow the patients and that there is less need for repeatedly checking in demographic details at different points of care delivery. This is of particular importance to community paediatricians, general practitioners, and health visitors when operating an immunisation campaign or screening service in ensuring maximum coverage.

\section{A thesaurus of clinical terms (Read codes)}

This is an extensive project involving over 50 speciality working groups of doctors, nurses, and professions allied to medicine who have the task of making lists of terms used in the clinical notes and assigning codes to these. It was first developed by a general practitioner, Dr James Read, and the codes have been adopted as the official NHS coding system administered by the Centre for Coding and Classification in Loughborough. It is able to incorporate all existing major coding systems (including ICD9, ICD10, OPCS, SNOMED, ICIDH). Members of the UK Child Health Specialty Working Group have made significant input into this project as well as producing a paediatric subset of ICD10 and have developed considerably the coding of diagnostic and non-diagnostic terms used in paediatric medicine, community child health, neonatology, and paediatric surgery. ${ }^{4}$ It is important for clinicians to realise that the terms included in the thesaurus are designed to be a reflection of those used in existing clinical records and include many non-diagnostic terms. If the system is going to be useful for clinical audit and research then agreed and unambiguous definitions of commonly used terms are needed, covering both administrative and clinical data. ${ }^{5}$ For example the terms 'speech delay' or 'NAI' would need further definition for the above purposes.

An interesting issue that requires clarification is who should do the coding of clinical notes? At present there are clinical coders employed by hospitals who allocate an ICD9 or alternative code to the diagnoses made by paediatricians in the notes as well as code the procedures that a child may undergo. Is this good enough and does it matter? Research has demonstrated that clinicians are better at coding the notes than clerical staff and that their participation in the process of coding leads to greater accuracy (over 30\% improvement), the benefit of case review, and creation of an accurate database for audit, research, and planning. ${ }^{6}$ Another study comparing clinician and clerical coding demonstrated differences in the length of stay recorded by the two groups and that the accuracy of diagnostic related groups, an often used indicator of resource utilisation, is very sensitive to the quality and consistency of the clinical coding data. ${ }^{7} \mathrm{~A}$ recent review of the fundamental weighted capitation formula (RAWP) used to allocate resources, some $£ 22$ billion, to regions, has emphasised the need to take into account the considerable variability of use of hospital care by patients with specific diagnoses or for specific procedures and the effects of social and geographic variables affecting resource utilisation. Although the authors recognised its importance, they were not given access to the diagnostic information collected from hospital episode statistics and used specialty groupings as a proxy. ${ }^{8}$

The vision of doctors coding on a routine basis is only likely to be feasible when there is a fully integrated electronic patient record and where the process of coding is eased by user friendly software. An electronic patient record in this context refers to a computerised record of the patient notes including diagnoses, procedures, treatment, drugs, results, and administrative details. A doctor would be able to write in clinical details, order investigations, read off results, order prescriptions, produce letters and discharge summaries, and carry out audit activity and research from the same work station electronically linked to the laboratories and pharmacy. The coding of clinical and administrative data in such a system would ideally be automatic.

\section{National standards for computer communications and a framework for security and confidentiality}

These are obviously vital components of such a strategy. The strategy sets up common standards and works towards NHS-wide networking so that information can be shared between health care professionals and organisations right across the nation. Many patients and clinicians are concerned about the possibility of breaches in personal confidentiality and there are strict rules guiding the use of data collected set out by the Data Protection Registrar.

The timetable for the above changes is ambitious. This year Read codes are being released in their new version. The Child Health Specialty Working Group are aiming to produce a paediatric subset of the Read codes by early 1995 with piloting having taken place in the autumn of 1994 . By July 1995, the new NHS number is to be issued, the national network spine should be in place, and $90 \%$ of 
general practices will have been computerised. By 1996 all major NHS organisations should be communicating electronically. By 1997, all community units are to have person based systems and by 2000 all hospitals are to have an integrated system in place. ${ }^{1}$

Several paediatricians have been involved with other aspects of NHS information strategy including some of the so called facilitating and pilot projects. These tend to have awkward acronyms sounding like physical signs or obscure investigations and include CISP (community information systems for providers), HISS (hospital information support systems), DISP (district information system for purchasers), ICWS (integrated clinical work station), CMDS (community contracting minimum data set), and PHIS (public health information systems) to name but a few. There has been a tradition of paediatric involvement with the National Child Health System which serves over two thirds of districts in one or other form as a community system. These projects are likely to develop further and many will move from pilot status to more widespread use and the fact that there has been paediatrician involvement will help to retain the child focus so easily lost when considering information systems.

\section{Some current issues in information strategy}

CONSULTATION/INTERNAL COMMUNICATION Part of the problem is keeping track with what seems like a whole informatics industry sprouting from the centre. The consultation documents for some of the projects are of 'War and Peace' proportions with unrealistic, tight deadlines for consultation. The British Paediatric Association have set up an interprofessional group of child health professionals including representation from nursing and professions allied to medicine to act as an 'expert group' to help advise members of our own professional organisations and to retain a child health viewpoint in Information Management Group strategy. This is particularly important in the area of community child health services at a time when the group appear only to recognise that child health medical services exist in hospitals or general practice settings.

CENTRAL VERSUS LOCAL GUIDANCE

In the current NHS, there is a question on how far will an independent trust feel they have to take heed of central guidelines. The investment in information technology cannot be underestimated and unless there are very explicit controls in place within the contracts with the commissioning bodies, then trusts are likely to go for a short term fix for commercial reasons. One of the issues facing paediatricians interested in the district-wide overview of the children's health is the difficulty in linking health events taking place in one provider unit with events occurring in another due to poor system communication and increasing commercial protectionism engendered by the internal market environment. A recent Audit Commission report has emphasised the need for better cooperation between providers of services to children especially those with complex social and health needs and the need to develop appropriate information systems and evaluation techniques to aid in this. ${ }^{9}$

DEVELOPING A MODEL OF SERVICE PROVISION Information system development is highly dependent on having a clear conceptual model of what the service looks like and it appears that the more doctors are involved in this dialogue with our informatics colleagues, the better the product. ${ }^{10}$ As a profession we have to work hard to develop our ideas of what we are trying to achieve in terms of explicit descriptions of the nature of interventions used and the outcomes we are aiming for, whether short or long term. We talk a lot about combined and integrated services but we spend less time talking about combined and integrated clinical guidelines or protocols of individual conditions. We cannot expect informatics to solve all our problems but if the NHS IM and $T$ strategy delivers what it promises we will have gone a long way in the right direction.

1 An information management and technology strategy for the NHS in England: getting better with information. NHS Management Executive Information Management Group, March 1993. (Produced by Central Office of Information; available from NHS ME.)

2 Windsor P. Introducing Körner - a critical guide to the work and recommendations of the steering group on health services information. BJHC Books, 1986.

3 Nissel M. People count - a history of the General Register Office. London: HMSO, 1989.

4 Walker D, Thomson K. Read clinical terms and child health. Arch Dis Child 1994; 71: 272-4.

5 Hutchison DCS. Making routine data adequate to support

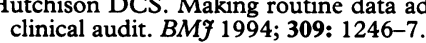

6 Yeoh C, Davies H. Clinical coding: completeness and Yeoh C, Davies H. Clinical coding: completeness and
accuracy when doctors take it on. $B M \mathcal{F}^{\prime} 1993 ; 306: 972-3$.

7 Walshe K, Harrison N, Renshaw M. Comparison of the quality of patient data collected by hospital and departmental computer systems. Health Trends 1993; 25: 105-8.

8 Carr-Hill RA, Sheldon TA, Smith P, Martin S, Peacock S, Hardman G. Allocating resources to health authorities; development of method for small area analysis of use of inpatient services. $B M F$ 1994; 309: 1046-9.

9 Audit Commission. Seen but not heard - coordinating community child health and social services for children in needexecutive summary. London: HMSO, 1994. 10 Lelliott P. Making clinical informatics work. BMF 1994;
308: 802-3. 